between those who had been on opposite sides of the street. This suggestion of air-borne infection in the open air seems difficult to credit. The operation of some other factor may be suspected, unidentifiable long after the event.

If this aspect of the disease remains obscure we have no doubt about its cause. Comprehensive laboratory studies disclosed no evidence of infection by any agent hitherto known to cause pneumonia. The positive results were obtained with specimens of lung from fatal cases. Films and cultures of these showed no significant bacteria, but of six guinea-pigs inoculated intraperitoneally with lung suspension four developed a febrile infection fatal in three to six days; one of these lungs was from a case of Broad Street pneumonia. Examination of peritoneal exudate, spleen, liver, and lung stained by silver impregnation showed numerous bacilli; an illustration shows these as varying in length and thickness with tapering ends; long threads were also present. This organism grew freely in the yolk sac of the chick embryo, but was non-pathogenic for mice. It was Gram-negative (by which is apparently meant that by Gram stain it was invisible) and non-acid-fast. It has since been cultivated on a common agar medium with supplements, but its colonial characteristics are not described.

Yolk-sac suspensions. were used for indirect fluorescence antibody tests. In these, dried films are treated first with dilutions of serum from a suspected case, and then with rabbit anti-human globulin serum conjugated with fluorescein; bacilli with which antibody has combined are then brightly stained. This test not only confirmed the presence of rising and high titre antibody in convalescent sera and its absence in control sera, but, thanks to the preservation of serum specimens in duplicate (acute and convalescent phase) from earlier epidemics and individuals, furnished proof that the disease is far from new. An epidemic in a mental hospital in the District of Columbia in 1965, with 89 cases and 12 deaths, was clearly of the same nature, and another in a county health department in Pontiac, Michigan, in 1968, with no deaths, was identified as a mild form of the disease. Oddly, these two as well as the Philadelphia outbreak occurred in the summer. Nine recently seen patients with sporadic pneumonia in six different states also showed seroconversion. This test has, of course, also been responsible for verifying the nature of recent cases in Spain and Britain.

Here, then, we have a new form of pneumonia, the source of the infection being unknown, and with apparently no characteristic clinical features. It may be suspected if no bacterium or virus can be found, but how can it be diagnosed short of sending serum to Atlanta? Doubtless cultures will come to Britain and enable us to perform the fluorescent antibody test here. But we should like to know whether a more direct method may be feasible. All studies of the bacillus seem to have been carried out so far with lungs from fatal cases. When further opportunities occur attention might well be directed to the sputum; presumably the silver impregnation method of staining which showed the organism in guinea-pigs has yet to be applied to this material. Even cultivation of the organism may be possible, for it has been achieved on a conventional medium. Apart from the question of diagnostic convenience, bacteriologists would dearly like to know much more about the bacillus itself; on present information it cannot be assigned to a genus or even classified more broadly. It also remains to be discovered where it exists between episodes of active infection.

\footnotetext{
1 British Medical fournal, 1977, 2, 1425.

${ }^{2}$ Fraser, D W, et al, New England fournal of Medicine, 1977, 297, 1189.

${ }^{3}$ McDade, J E, et al, New England Fournal of Medicine, 1977, 297, 1197.
}

\section{Coeliac disease in Galway}

Our definition of coeliac disease still depends on the response to treatment. A coeliac is someone whose small-intestinal mucosa is genetically predisposed to damage from exposure to gluten. The abnormality persists throughout life, unlike the usually less severe lesion of transient gluten intolerance found in some children. ${ }^{2}$ The intestinal damage should be shown to improve substantially after gluten withdrawal. When the diagnosis is still in doubt, a third intestinal biopsy may be needed to show that the damage recurs after a gluten challenge.

Participants at the Third International Coeliac Symposium held recently in the beautiful setting of Galway seemed agreed on these points. Nevertheless, the genetic pattern, the toxic fraction of gluten, the diagnostic mucosal lesion, and the criteria for response to gluten withdrawal and to gluten challenge all remain undefined and open to debate. Such discussion is the meat and drink of symposia and there was plenty of all three ingredients.

The histocompatibility antigens HLA B8 and especially HLA DW3 are associated with coeliac disease. ${ }^{34}$ An even closer association seems to be found with a specific B-lymphocyte-associated antigen. ${ }^{5}$ As Strober and his colleagues from Bethesda suggested, there may be two genes coding for proteins which make up a single structure on the surface of B cells. Such a structure might be a receptor for gliadin, the toxic fraction of gluten.

The meeting was reminded by the workers from Leiden, where the important Second International Symposium was held in 1974, that gliadin itself is a heterogeneous protein currently defined by its electrophoretic mobility. It takes a great deal of time to obtain electrophoretically pure alphagliadin (which probably contains the toxic subfraction of gliadin) in sufficient quantities to use in clinical tests. Different centres use their own schedules for challenging the mucosa of the treated patient with coeliac disease. Most still use gluten, but the amount, presentation, and duration of the gluten challenge all vary. The symposium set up a small working group to try to agree a standard technique. One aspect of gluten challenge that provoked interest in physicians caring for adults was the different response of the treated coeliac child's mucosa to gluten. If the child remains symptom free on return to a normal diet re-exposure to gluten may be prolonged for two years or more before it induces significant mucosal damage. In contrast, changes can be induced in adult coeliacs given 20-30 g gluten or a few days' normal diet (containing about $12 \mathrm{~g}$ gluten daily). Some adult patients can hardly tolerate even this amount of gluten without gastrointestinal symptoms.

High hopes were raised by some elegant work on a variant of Chinese Spring wheat which lacks alpha-gliadins and hence could potentially be eaten by these patients. Unfortunately, predictions were not supported by evidence from intestinal biopsy studies, which alone could show whether patients can eat this wheat with impunity. The results of the home team's study of "detoxified" gliadin with a carbohydrate moiety removed also ran into difficulties at the conference, for many thought that the small biopsy specimens shown as evidence of lack of damage after challenge with this substance seemed to contain appreciable numbers of abnormal epithelial surface cells. By the end of the ensuing discussion the case for detoxification depended largely on the findings in one patient, and the verdict remained "not proven." 
The basis for the mucosal immune response to gluten seems likely to be the precipitation of antigen-antibody complexes in the small intestinal mucosa with complement activation. ${ }^{6}$ IgG, IgM, and complement may all be implicated in the persisting, long-term damage. Some interesting work on skin testing for diagnostic screening, from Oxford ${ }^{7}$ and Bristol, ${ }^{8}$ suggested that in time we may be able to reduce the numbers of intestinal biopsies needed for diagnosis-one of the aims of the Coeliac Society, which sponsored the Oxford work.

We still have to agree on how abnormal the untreated mucosa should be to qualify for the diagnosis of coeliac disease, how much improvement should result from gluten withdrawal, and how much damage should follow gluten challenge. Finally, what about the "unresponsive coeliac"? A lack of response is uncommon so long as a patient continues his gluten-free diet strictly, but there are increasing reports of adult patients who have responded clinically and morphologically to gluten withdrawal and who then, after a lapse from the diet, have become unresponsive to an apparently strict regimen. ${ }^{9}$ Some of these patients can be tided over an unresponsive period, but this complication of coeliac disease merits more study.

1 Walker-Smith, J A, Archives of Disease in Childhood, 1970, 45, 523

2 Walker-Smith, J A, Archives of Disease in Childhood, 1972, 47, 155

${ }^{3}$ Falchuk, Z M, Rogentine, G M, and Strober, W, fournal of Clinical Investigation, 1972, 51, 1602.

- Keuning, J J, et al, Lancet, 1976, 1, 506.

5 Mann, D L, et al, Lancet, 1976, 1, 110

${ }^{6}$ Doe, W F, Henry, K, and Booth, C C, in Proceedings of the Second International Coeliac Symposium, eds W Th J M Hekkens and A S Pena, p 189. Leiden, Stenfert Kroese, 1974.

7 Anand, B S, Truelove, S C, and Offord, R E, Lancet, 1977, 1, 118.

${ }^{8}$ Baker, P G, and Read, A E, Quarterly fournal of Medicine, 1976, 45, 603.

o Neale, G, British Medical fournal, 1968, 2, 678.

\section{Small-bowel ischaemia and the contraceptive pill}

Among the many cardiovascular complications of the contraceptive pill one of the least familiar is small-bowel ischaemia -but one associated with a high mortality rate and much morbidity. Hoyle et $a l^{1}$ have recently reviewed 21 cases and found that half the patients had died and half had required two or more operations, resulting in the removal of much of the small bowel.

Small-bowel ischaemia occurs in women taking the pill as a result of either mesenteric artery or mesenteric vein thrombosis. Arterial thrombosis carries nearly twice the mortality of venous thrombosis but is only half as common. The dosage of oestrogen seems to be an important factor: nine of the women reviewed by Hoyle $e t \mathrm{al}^{1}$ had been taking $0.5 \mathrm{mg}$ daily, while the remaining 12 women had been taking a higher dose. Over half the women had been taking an oral contraceptive for a year or more, but two were only in their first cycle. In Hoyle's personal series of three patients all were of blood group A rhesus positive-a group already shown to predispose to thrombosis..$^{2-4}$ All three were smokers and two were hypertensive. Again, the association of hypertension and blood group A has been reported in this condition. ${ }^{5}$

Ischaemic colitis, but transient, has also been reported in young women taking oral contraceptives. ${ }^{6-8}$ In these cases the patients had no predisposing disorders and presented with the classical symptoms of abdominal pain and bloody diarrhoea. Diagnosis was based on the clinical picture and the appearances on barium enema-a narrowed segment of colon and thumb-printing of the mucosa. In these patients stopping the use of oral contraceptives allowed the bowel to recover, and later barium enema examinations showed normal results.

In contrast, the symptoms of small-bowel ischaemia are both more varied and vague, and the diagnosis is often delayed. The dominant presenting symptom in small-bowel ischaemia, found in all patients, is abdominal pain. In over one-quarter of the reported cases the pain had been present for two or more weeks, suggesting a slow onset. Some patients had associated nausea and vomiting, while others complained of diarrhoea. Occasional patients have had a haematemesis or melaena, depending on the level of the ischaemic segment. On examination the patient has usually been found to be febrile with generalised abdominal tenderness. Bowel sounds are present unless infarction has occurred, in which case there is rebound tenderness. Haematological investigations may show a raised white count and increased serum fibrinogen and fibrinogen degradation products. On barium meal and follow through there may be a narrowed segment of small bowel. ${ }^{9}$ The place of ${ }^{131} \mathrm{I}$ uptake and angiography in the diagnosis of this condition has yet to be established.

Unfortunately, in nearly all the cases reported the diagnosis has been made only at laparotomy, when the bowel was usually infarcted. The fact that many of the patients had had pain for two or more weeks suggests that the condition might well be reversible if it could be detected earlier. A diagnosis of smallbowel ischaemia should be carefully considered in any woman taking an oral contraceptive who presents with vague abdominal pain and has an associated condition known to predispose to circulatory disorders: cigarette smoking, hyperlipidaemia, diabetes, hypertension, obesity, ${ }^{10}$ or blood group A. If small-bowel ischaemia seems the likely diagnosis the contraceptive pill should be stopped immediately and treatment started with heparin. In this way the mesenteric thrombosis might be arrested before ischaemia becomes irreversible.

1 Hoyle, M, et al, British fournal of Surgery, 1977, 64, 533.

2 Jick, R, et al, Lancet, 1977, 1, 539.

3 Fagerhol, M K, Abildgaard, V, and Kornstad, L, Lancet, 197.1, 2, 664

4 Mourant, A E, Kopec, A C, and Domaniewsha-Sobczak, S K, Lancet, $1971,1,223$.

${ }^{5}$ Lowry, J B, Orr, K G, and Wade, W G, Fournal of the Irish Medical Association, 1969, 62, 260.

- Kilpatrick, Z M, et al, New England fournal of Medicine, 1968, 278, 438

7 Hurwitz, R L, et al, Annals of Surgery, 1970, 172, 892.

${ }^{8}$ Cotton, P B; and Thomas, M L, British Medical fournal, 1971, 3, 27.

${ }^{9}$ Nothmann, B J, Chittinand, S, and Schuster, M M, American fournal of Digestive Diseases, 1973, 18, 361.

10 Mann, J I, et al, British Medical fournal, 1975, 2, 241.

\section{Flap lacerations}

Any experienced casualty surgeon knows that the combination of a V shaped wound on the shin and an elderly woman usually indicates trouble ahead. It is likely to take a long time to heal, and the outlook will be worse if the flap is hinged at its distal edge. Why should these lesions be so troublesome? The primary factors are the thin skin, loss of tissue vitality, dependent position, and slower healing time in the leg, while a distally based flap further exaggerates the difficulties of blood supply and venous drainage. The final insult may be 